\title{
Data Hiding in Halftone Images by Stochastic Error Diffusion
}

\author{
Ming Sun Fu, Oscar C. Au \\ Department of Electrical and Electronic Engineering, \\ Hong Kong University of Science and Technology, \\ Clear Water Bay, Hong Kong, China. \\ Tel: +852 2358-7053, Fax: +852 2358-1485, Email: fmsun@ust.hk, eeau@ust.hk
}

\begin{abstract}
In this paper, we propose a novel method called DHSED to hide binary visual patterns in two error diffused halftone images. While one halftone image is only a regular error diffused image, stochastic error diffusion is applied to the other image to generate special stochastic characteristics with respect to the first image such that the visual pattern would appear when the two halftone images are overlaid Simulation results show that the two halftone images have good visual quality, and the hidden pattern appears with "normal" and "lower-than-normal" intensity when the two halftone images are overlaid.
\end{abstract}

\section{I ntroduction}

Image data hiding is the hiding or embedding of invisible data in an image without affecting its perceptual quality such that the hidden data can be extracted with some procedure. The study of data hiding techniques is commonly called steganography [2]. Major applications of data hiding include the embedding of secret messages and data. In particular, the embedded data can be the metadata in MPEG7 applications. In recent years, two important subclasses of data hiding methods are fragile watermarks and robust watermarks. Fragile watermarks [1] are designed to be broken easily by common image processing operations. The broken watermark serves as an indication of alteration of the original image and is useful for authentication. Major applications include tampering detection of images placed on the World Wide Web and authentication of images received from questionable sources. Robust watermarks [1] are required to remain in the watermarked image even after it has been attacked by attackers or processed by common image processing operations such as filtering, requantization, scaling, cropping, etc. Major applications include copyright protection, distribution and copy control, etc.

In this paper, we are concern about data-hiding for halftone images. Halftone images contain only 2 tones and are generated by a procedure called halftoning from multi-tone images. Although there are only 2 tones, halftone images look like the original multi-tone images when viewed from a distance.
Halftone images are widely used in the printing of books, magazines, newspapers and in computer printers. It is often desirable to hide visual patterns within the printed halftone images such that the hidden patterns can be viewed when some appropriate image is overlaid. Some visual pattern examples include short messages with large font size, logos, graphics or clip arts which can be used for authentication, and conveying secret messages or product related information.

However, there are still some existing techniques for halftone image data hiding. They can be divided into two classes. One class of techniques embed invisible digital data into halftone images such that the data can be read by scanning the halftone images and applying some extraction algorithms on the scanned image. Some used two different dithering matrices for the ordered dithering halftone generation [5] such that the different statistical properties due to the two dithering matrices can be detected in the future. Some embedded data in the angular orientation of circularly asymmetric halftone dot patterns that were written into the halftone cells of digital halftone images [6]. Some hid data at pseudo-random locations in ordered dithered and error diffused images by self toggling or pair toggling $[7,8]$ of the halftone values. Some hid data in error diffused images using error diffusion to mitigate the distortion due to the data hiding, resulting in good visual quality $[7,9]$. In these techniques, although the hidden data can be images also, the algorithms do not provide for viewing the hidden images directly on the halftone images.

A second class of halftone image watermarking techniques embed hidden visual patterns into two or more halftone images such that when the two images are overlaid, the hidden image can be viewed directly on the halftone images. The hidden data must be visual patterns and they are meant to be visually inspected on the halftone images. Some used stochastic screen patterns [10] and conjugate halftone screens [11] to embed the hidden patterns in ordered dithered halftone images. Due to the conjugate properties, when the two ordered dithered images are overlapped, the hidden visual patterns appeared as dark patterns on the halftone image. However, we are 
not aware of any existing methods for error diffused images.

In section 2, we propose a novel algorithm called Data Hiding by Stochastic Error Diffusion (DHSED) to embed hidden visual patterns in two or more error diffused halftone images such that the hidden patterns can be visually inspected when the images are overlaid. In Section 3, simulation results are shown.

\section{Data Hiding by Stochastic Error Diffusion (DHSED)}

In this section, we propose a novel algorithm called Data Hiding by Stochastic Error Diffusion (DHSED) to hide invisible watermarking data or patterns in two related error diffused halftone images such that the hidden patterns can be visually detected when the images are overlaid.

DHSED uses two $M \times N$ halftone images, $Y_{0}$ and $Y_{1}$, to hide some binary image $H$ which can be visually detected by overlaying $Y_{0}$ and $Y_{1}$. Let $X$ be the original $M \times N$ multitone image from which $Y_{0}$ and $Y_{1}$ are obtained. We will use $x(i, j)$ and $y_{i}(i, j)$ to represent the pixels at location $(i, j)$ of $X$ and $Y_{i}$ respectively. In general, all quantities associated with $Y_{i}$ would have a subscript $i$. The hidden binary pattern $H$ is assumed to have the same size $M \times N$ as $Y_{0}$ and $Y_{1}$. In the case that the desirable hidden patterns have size smaller than $M \times N$, they can be enlarged and padded to $M \times N$. The black pixels in $H$ can carry meaningful foreground patterns such as text, logo, graphics or clip arts while the white pixels can carry the background. Neither the foreground nor background patterns should contain very fine features because DHSED tends not to be effective in retaining fine features.

The first image $Y_{0}$ is generated by regular error diffusion with no hidden visual patterns. In other words, at each pixel location $(i, j)$, a value $f_{0}(i, j)$ related to the current multitone pixel value $x(i, j)$ is compared with a threshold $T(T=128)$. And $y_{0}(i, j)$ will be 0 if $f_{0}(i, j)<T$ and 255 if $f_{0}(i, j) \geq T$. The error $e_{0}(i, j)$ is fed forward or diffused to the future pixels by a causal kernel. Note that $e_{0}(i, j)$ is not the difference of $x(i, j)$ and $y_{0}(i, j)$, but the difference of $f_{0}(i, j)$ and $y_{0}(i, j)$. Suppose the causal kernel is the Jarvis kernel in Fig. 1. The $f_{0}(i, j)$ is the sum of the current pixel value $x(i, j)$ and the feed-forward error $a_{0}(i, j)$ as follows.

$$
\begin{aligned}
& a_{0}(i, j)=\frac{1}{48} \sum_{k, l=-2}^{2} e_{0}(i+k, j+l) *_{w}(k, l) \\
& f_{0}(i, j)=x(i, j)+a_{0}(i, j) \\
& y_{0}(i, j)=\left\{\begin{array}{cc}
0 & f_{0}(i, j)<T \\
255 & \text { otherwise }
\end{array}\right. \\
& e_{0}(i, j)=f_{0}(i, j)-y_{0}(i, j)
\end{aligned}
$$

The second image $Y_{1}$ is generated by applying Stochastic Error Diffusion (SED) to $X$ with respect to $Y_{0}$ to hide $H$. In SED, the hidden binary image $H$ is used to turn on or off the stochastic properties on a pixel-by-pixel basis. Let $H_{B}$ be the collection of the locations of all the black pixels in $H$. Similarly, let $H_{W}$ be the collection of all the white pixel locations in $H$. For $(i, j) \in H_{W}$, the pixel $y_{1}(i, j)$ in $Y_{1}$ is forced to be identical to the co-located pixel $y_{0}(i, j)$ in $Y_{0}$. In other words, $y_{1}(i, j)=y_{0}(i, j)$ for $(i, j) \in H_{W}$. For $H_{B}$, error diffusion is applied with some special boundary conditions using the same error diffusion kernel as in $Y_{0}$ such that the texture and the look-and-feel of the regions in $Y_{1}$ are very similar to, if not the same as, the corresponding regions in $Y_{0}$. The purpose of the special boundary conditions is to create a difference in the "phase" of error diffusion texture in $Y_{1}$ relative to that in $Y_{0}$ such that the collocated pixels in the $Y_{0}$ and $Y_{1}$ can be considered as statistically independent.

Firstly, morphological dilation with some structuring element $S$ is applied to $H_{B}$ to give $C=H_{B} \oplus S$. If $S$ is a $(2 L+1) \times(2 L+1)$ square matrix with current pixel being in the center, $C$ is basically $H_{B}$ expanded outward both horizontally and vertically by $\mathrm{L}$ pixel. An example is shown in Fig. 2 in which $H_{B}$ is a single connected region. Define region $D=C \cap H_{B}{ }^{c}=C \cap H_{W}$, which is the dark region outside $H_{B}$ in Fig. 1. In SED, the boundary condition is that the error $e_{1}(i, j)$ is assumed to be zero outside $C$, i.e. $e_{1}(i, j)=0$ for $(i, j) \notin C$. For $(i, j) \in D$, Eqns. 1 and 2 are applied to compute $a_{1}(i, j)$ and $f_{1}(i, j)$ using the neighboring $e_{1}$. Eqns. 3 and 4 are not applied. Instead of Eqn. 3, we set $y_{1}(i, j)=y_{0}(i, j)$ because $D \subset H_{W}$. Instead of Eqn. 4, we apply Eqn. 5

$$
e_{1}(i, j)=\max \left(\min \left(f_{1}(i, j)-y_{1}(i, j), 127\right),-127\right)
$$


which is basically Eqn. 4 with clipping. For $(i, j) \in H_{B}$, regular error diffusion (i.e. Eqns. 1 to 4 with subscript changed from 0 to 1 ) is applied. In this way, region $H_{B}$ will have the characteristic texture of the regular error diffusion. But the phase of the error diffusion texture in region $H_{B}$ of $Y_{1}$ would be different from the corresponding region of $Y_{0}$.

When $Y_{0}$ and $Y_{1}$ are overlaid, the regions corresponding to the white regions of $H$ should have normal intensity while those corresponding to the black regions of $H$ should have lower-than-normal intensity.

\section{Simulation Results}

The proposed DHSED is simulated using the $512 \times 512$ Lena image. The error diffused halftone image using Jarvis kernel with no data embedded, or $Y_{0}$, is shown in Fig. 3. In this experiment, the hidden pattern $H$ contains 3 rows of "UST" as shown in Fig. 5. The image $Y_{1}$ with $H$ embedded is shown in Fig. 4. With distortion due to the hidden $H$, the $Y_{1}$ has good, though not perfect, subjective visual quality compared with $Y_{0}$. Some minor trace of the edges of $H$ can be seen in $Y_{1}$. When $Y_{0}$ and $Y_{1}$ are overlaid in Fig. 6, the hidden image is revealed in the halftone image. The regions corresponding to the white regions of $H$ have "normal" intensity in Fig. 6. The regions corresponding to the dark regions of $H$ have "lowerthan-normal" intensity. Consequently, the difference in intensity reveals the hidden pattern $H$ in the halftone image.

The contrast of the hidden pattern is not uniform throughout the whole image. The contrast is not good in bright regions such as the top "S" on the bright hat region and part of the top " $T$ " on the bright background region. It is also poor in high-texture regions such as the middle " $U$ " and bottom " $U$ " on the Lena's feathery hat, and the middle " $S$ " on Lena's eye. The contrast is good in the rest of the image. In general, the contrast is good in smooth regions with mid-gray brightness such as Lena's shoulder.

\section{Conclusion}

In this paper, we propose a novel method called Data Hiding Stochastic Error Diffusion (DHSED) to hide binary visual patterns in two error-diffused halftone images. Simulation results show that the halftone images with the embedded visual patterns retain good visual quality. When the two halftone images are overlaid, the hidden visual pattern is revealed on the halftone image. The contrast is good in smooth regions with mid-gray brightness.

\section{References}

[1] F. Mintzer, et al., "Effective and Ineffective Digital Watermarks", Proc. of IEEE Int. Conf. on Image Processing, Vol. 3, pp. 913, Oct. 1997.

[2] N.F. Johnson, S. Jajodia, "Exploring Steganography: Seeing the Unseen", IEEE Computer, Vol.31, No.2, pp.26-34, Feb. 1998.

[3] B. E. Bayers, "An Optimum Method for Two Level Rendition of Continuous Tone Pictures," Proc. of IEEE Int. Communication Conf., pp26112615, 1973.

[4] R.W. Floyd, L. Steinberg, "An Adaptive Algorithm for Spatial Grayscale," Proc. SID, pp. 75-77, 1976.

[5] Z. Baharav, D. Shaked, "Watermarking of Dither Halftoned Images", Proc. of SPIE Security and Watermarking of Multimedia Contents, pp. 307313, Jan 1999.

[6] R. T. Tow, "Methods and Means for Embedding Machine Readable Digital Data in Halftone Images", United States Patent Number 5,315,098

[7] M.S. Fu, O.C. Au, "Data Hiding in Halftone Image by Pixel Toggling", Proc. Of SPIE Int. Conf. On Security and Watermarking of Multimedia Contents, Vol. 3971, pp. 228-236, Jan 2000.

[8] M.S. Sun, O.C. Au, "Data Hiding by Smart Pair Toggling for Halftone Images", Proc. Of IEEE Int Conf. On Acoustics, Speech and Signal Processing, Vol. 4, pp. 2318-2321, Jun. 2000.

[9] M.S. Sun, O.C. Au, "Modified Data Hiding Error Diffusion for Image Halftoning', Proc. Of SPIE Conf. On Visual Communication and Image Processing, Vol. 3, pp. 1671-1680, Jun 2000.

[10] K.T. Knox, "Digital Watermarking Using Stochastic Screen Patterns", United States Patent Number 5,734,752.

[11]S. G. Wang, "Digital Watermarking Using Conjugate Halftone Screens", United States Patent Number 5,790,703. 


\begin{tabular}{|c|c|c|c|c|}
\hline & & $(0,0)$ & 7 & 5 \\
\hline 3 & 5 & 7 & 5 & 3 \\
\hline 1 & 3 & 5 & 3 & 1 \\
\hline
\end{tabular}

Figure 1 Jarvis error diffusion kernel

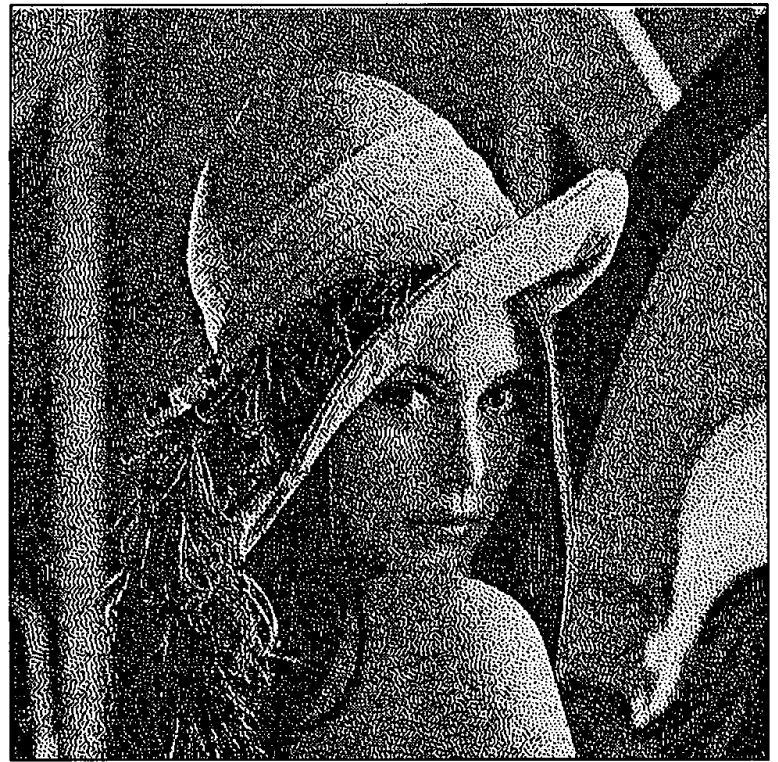

Fig. $3 Y_{0}$ from 512x512 Lena with nothing hidden

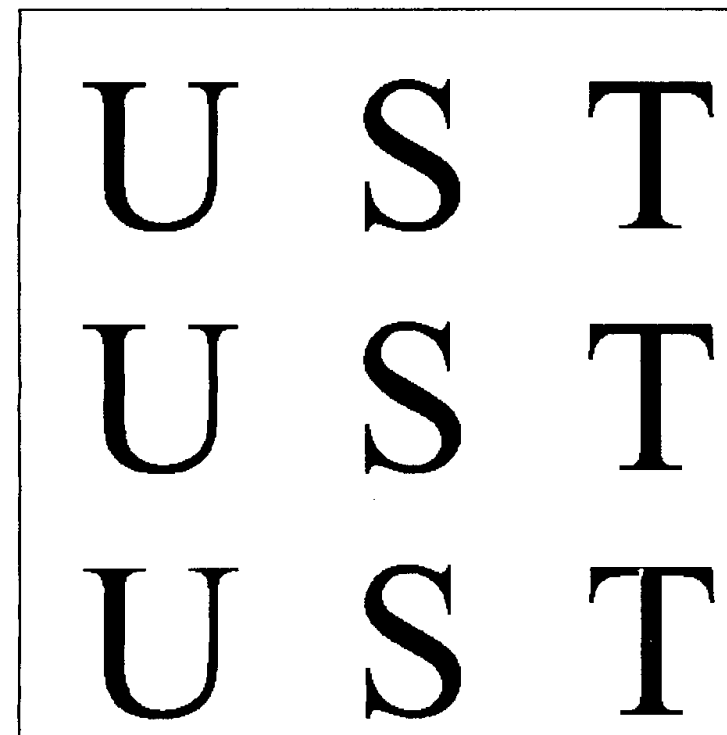

Fig. $5 H$ to be hidden
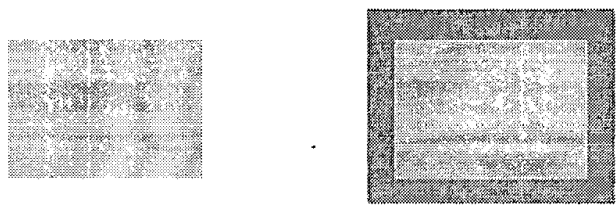

Fig. 2 An example of (left) $H_{B}$; (right) $C=H_{B} \oplus S$ with dark region being $D=C \cap H_{B}{ }^{c}$

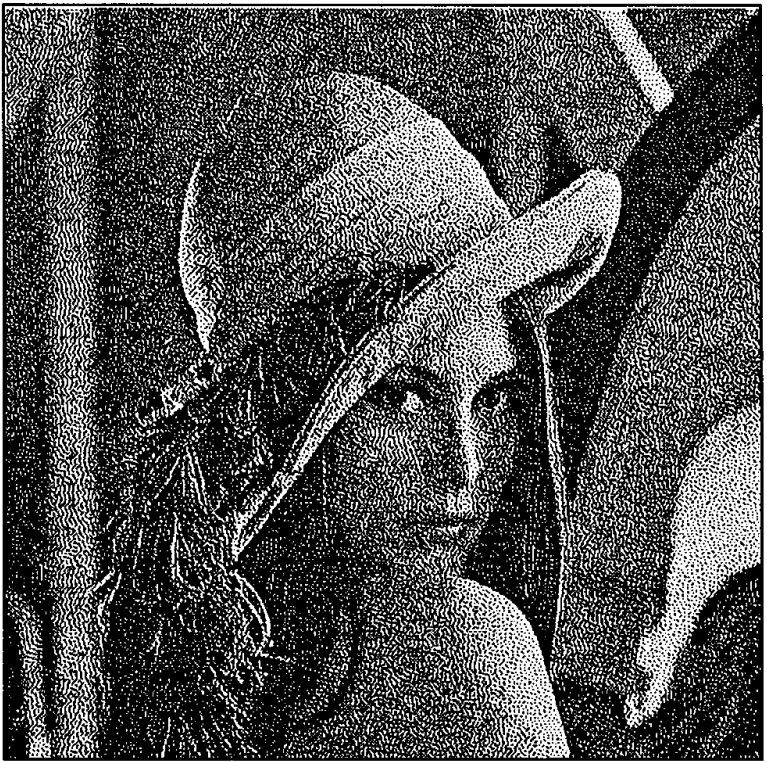

Fig. $4 Y_{I}$ with $H$ hidden

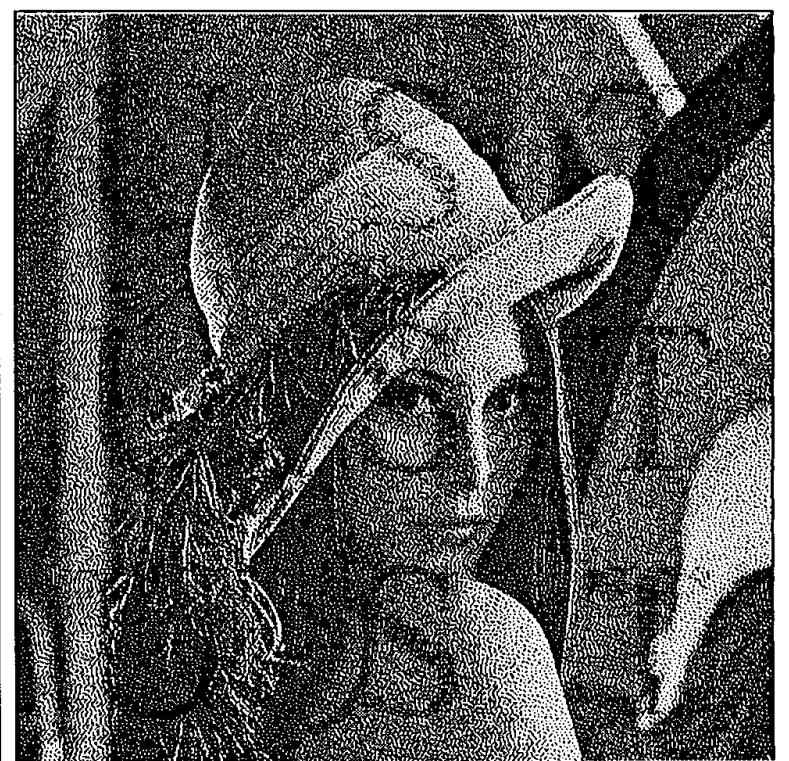

Fig. $6 Y_{0}$ and $Y_{l}$ overlaid showing the hidden $H$ 